\title{
A MATERIAL EXPERIMENT FOR SMALL SATELLITES TO CHARACTERISE THE BEHAVIOUR OF CARBON NANOTUBES IN SPACE - DEVELOPMENT AND GROUND VALIDATION
}

\author{
Elisabeth Abbe $^{(1)}$, Thomas Renger ${ }^{(2)}$, Maciej Sznajder ${ }^{(2)}$, Benjamin Klemmed $^{(3)}$, Elisa Sachse ${ }^{(4)}$, René Hübner $^{(5)}$, \\ Tilman Schüler $^{(1)}$, Yves Bärtling ${ }^{(1)}$, Benjamin Muchow ${ }^{(1)}$, Martin Tajmar $^{(1)}$, Tino Schmiel $^{(1)}$ \\ (I) Institute of Aerospace Engineering, Technische Universität Dresden, Germany, Marschnerstr. 32, \\ 01307 Dresden, \\ elisabeth.abbe/tilman.schueler/yves.baertling/benjamin.muchow/martin.tajmar/tino.schmiel \\ @ tu-dresden.de
}

(2) DLR Institute for Space Systems, Mechanics and Thermal Systems, Robert Hooke Str. 7, 28359 Bremen, Germany, thomas.renger/maciej.sznajder@dlr.de

(3) Physical Chemistry, Technische Universität Dresden, Germany, Bergstraße 66b, 01062 Dresden, benjamin.klemmed@tu-dresden.de

(4) Institute for Material and Beam Technology IWS, Fraunhofer Dresden, Germany, Winterbergstr. 28, 01277 Dresden, elisa.starruss@iws.fraunhofer.de

(5) Institute of Ion Beam Physics and Materials Research, Helmholtz-Zentrum Dresden-Rossendorf, Germany, Bautzner Landstraße 400, 01328 Dresden, r.huebner@hzdr.de

\begin{abstract}
Over the last years, Carbon Nanotubes (CNT) drew interdisciplinary attention. Regarding space technologies a variety of potential applications were proposed and investigated. However, no complex data on the behaviour and degradation process of carbon nanotubes under space environment exist. Therefore, it is necessary to investigate the performance of these new materials in space environment and to revaluate the application potential of CNTs in space technologies.

Hence, CiREX (Carbon Nanotubes - Resistance Experiment) was developed as a part of a student project. It is a small and compact experiment, which is designed for CubeSat class space satellites. These are a class of nanosatellites with a standardized size and shape. The CiREX design, electrical measurements and the satellites interfaces will be discussed in detail. CiREX is the first in-situ space material experiment for CNTs.

To evaluate the data obtained from CiREX, ground validation tests are mandatory. As part of an extensive test series the behaviour of CNTs under solar ultra violet light (UV) and vacuum ultraviolet light (VUV) was examined. Singlewalled carbon nanotubes (SWNT), multi-walled carbon nanotubes (MWNT) and MWNT/resin composite (ME) were exposed to different light sources. After the exposure, the defect density was investigated with Raman spectroscopy. There is a clear indication that UV and VUV light can increase the defect density of untreated CNTs and influencing the electrical behavior.
\end{abstract}

KEYWORDS Space Environment, Material Experiment, CubeSat, Carbon Nanotubes, Electrical Behaviour, Solar light

\section{INTRODUCTION}

With the continually increasing attention on CNTs, a lot of potential applications have been proposed over the last years [1,2]. Even regarding space technologies, a large number of new applications has been developed e.g. electrostatic discharge coatings, electromagnetic shields or high-strength structural materials [3, 4]. However, the majority of these applications is still in an early stage of development.

Only a limited number of real space experiments and applications of CNTs exists nowadays. As the very first organisation, the NASA used CNT-based elements for the space mission JUNO to Jupiter. The main function of those elements is the protection against electro-static discharge. These new elements meant to replace metallic solutions bonded to composites [3], but currently there is no additional information about this mission available.

One of the most important space material experiments for carbon nanotubes is MISSE-8 [5]. Here, CNT-yarns were exposed to the space environment on the ram- and wake- side of the International Space Station. However, there was no in situ monitoring during the experiments. Thus, all samples were examined after their return to earth, with the CNTs exhibiting an increased resistance and tensile strength [5]. Overall, MISSE-8 does not provide any information about 
the material behaviour during different orbits and the potential influence of the space environment. Furthermore, material property changes could occur during return to Earth (e.g. healing processes).

Despite first insights, a complex understanding of the material behaviour and degradation processes in space environment is still missing. Only a few number of articles about proton and electron irradiation were published recently [6]-[9]. Furthermore, a few groups tested the behaviour of CNTs under UV or Gamma irradiation [10]-[12]. All these studies used different materials, measurement strategies, preparation techniques, and test parameters. Because of the fact that CNTs are strongly influenced by their surrounding environment, it is not possible to transfer these first results to potential applications in space. Therefore, it is necessary to design an experiment, which evaluates the influence of the space environment to revaluate and optimise these technologies.

Hence, we developed a small experiment testing CNTs in CubeSats. CiREX is an in-situ space experiment, which allows observing the resistance of carbon nanotube networks in the complex space environment. These results have to be verified through ground validation tests. For this test series, we investigated the influence of proton [9], electron and gamma irradiation on carbon nanotubes. As part of the test series, the electrical behaviour under solar light was investigated. Most of the published studies about electromagnetic radiation are focused on specific wavelengths. Mikò et al. reported on the behaviour of CNT bundles under irradiation at a wavelength of $365 \mathrm{~nm}$ [13]. Grujicic et al. did provide computational studies on the oxidation of CNTs applying a light source of $240 \mathrm{~nm}$ [10]. In this work, we studied the influence of solar light within a wavelength range of $40 \mathrm{~nm}$ up to $2150 \mathrm{~nm}$. The aim of these investigations is to demonstrate the functionality of CiREX using solar irradiation as an example and to understand the degradation behaviour of the material samples under solar radiation.

\section{CiREX ON SOMP2 (STUDENT ON-ORBIT MEASUREMENT PROJECT2)}

\subsection{Mechanical and electrical design of CiREX}

The electrical properties for MWNT and SWNT are extraordinary. Because of the almost one-dimensional structure, the electrical transport is ballistic, which only applies within the tubes. In CNT networks the contacts between tubes become effective and the electrical resistance increases. Adsorbed ions and functionalized groups can act as scattering centres, which influence the charge flow in the network. Therefore, an ohmic behaviour of the test samples can be ascertained. Consequently, the ohmic behaviour of CNT networks is strongly influenced by adsorbed ions and molecules (1), the defect structure and the contact resistance of the network (2) and thermal modifications (3).

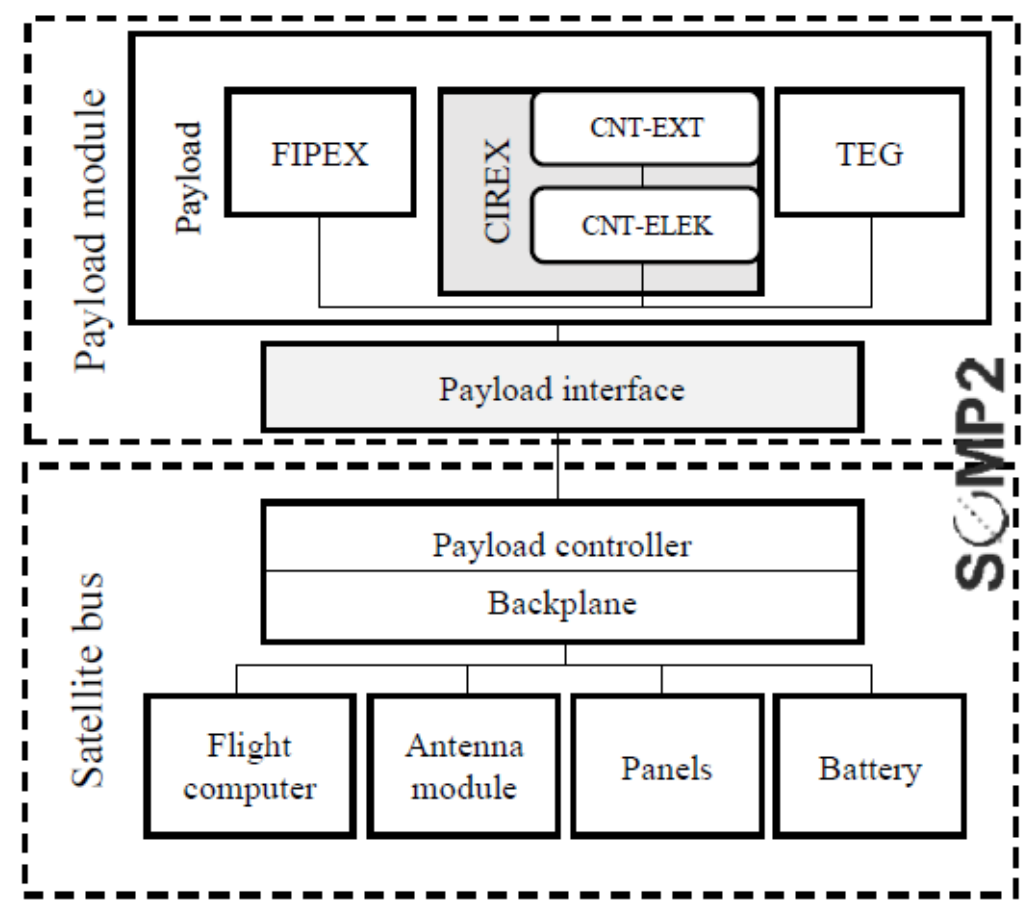

Figure 1: Design and electrical Interface of SOMP2 (Student On-Orbit Measurement Project) with CiREX consisting of the sensor module (CNT-EXT) and the electrical circuit board (CNT-ELEK). 
The influence of these effects was considered during the design process of CiREX. It is a small and simple experiment, which was designed to measure the electrical resistance of carbon nanotube networks in space. CiREX consists of two different parts: the sensor module (CNT-EXT) and the electrical circuit board (CNT-ELEK) (see Figure 1).

Both parts are designed to fit in a CubeSat layout. The CubeSat standard exists since 1999 and defines a cubic unit of 10x10x10 $\mathrm{cm}^{3}$ with a mass of less than $1.33 \mathrm{~kg}$ per unit. CiREX was developed for SOMP2 (Student On-Orbit Measurement Project), which is a double-unit CubeSat.

CNT-EXT includes ten material samples: four samples of multi-walled carbon nanotube composite (ME), three singlewalled carbon nanotubes (SW) and three multi-walled carbon nanotubes (MW) samples. The CNTs and the gold contact pads are printed on an $\mathrm{Al}_{2} \mathrm{O}_{3}$ substrate (details see section 2.2). These substrates are mounted on a three-dimensional circuit board and chip bonds provide a contact between the material sample and circuit board. To protect the bonds during the launch against vibrations, they are stabilized with silicone (NuSil CV-1152). In addition to the material samples, CNT-EXT contains a temperature sensor and a photodiode (see Fig. 2). Two of the ten material samples are shielded against low-energy irradiation and direct solar radiation through an aluminium cover. The thickness of the cover amounts $1 \mathrm{~mm}$.

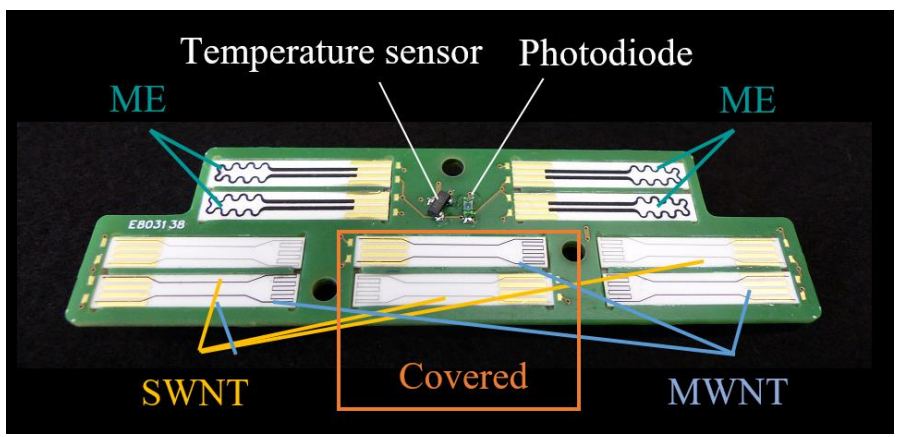

Figure 2: CNT-EXT without cover plate.

CiREX is well integrated in the payload structure of SOMP2. CNT-EXT is embedded in the front panel. This allows a free angle of nearly $180^{\circ}$ for the material samples. Hence, the CNT samples are directly exposed to space next to the Flux-(Phi)-Probe-Experiment (FIPEX). FIPEX consist of two solid state electrolyte sensor units, which measure the partial pressure of atomic oxygen and distinguish atomic oxygen from molecular oxygen. FIPEX requires a pointing accuracy of $\pm 10^{\circ}$ and a pointing knowledge of $\pm 2^{\circ}$. Therefore, the position data over the lifetime from SOMP2 are well known. Besides FIPEX, the Thermoelectric-Generator-Experiment (TEG) is another important payload. TEG utilizes the heat flux through thermoelectric devices to generated power. Hence, the thermal conditions inside the satellite are well known.

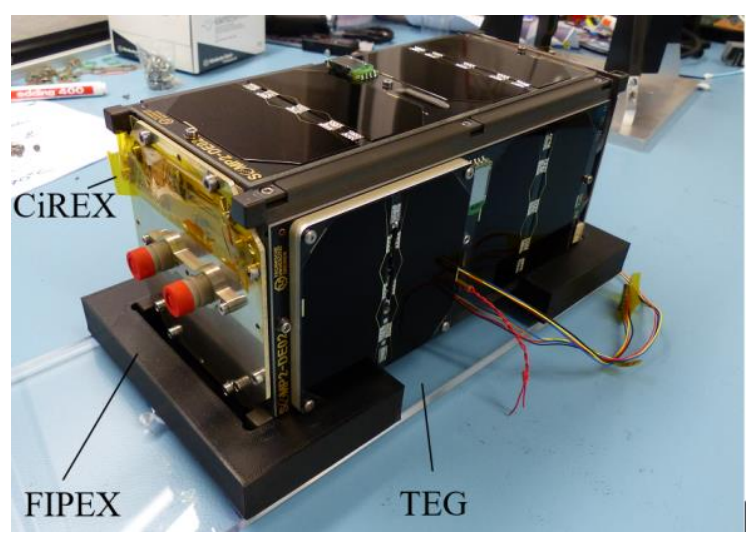

Figure 3: CiREX, FIPEX and TEG integrated in SOMP2.

To protect the satellite and CNT-ELEK, the rear side of CNT-EXT is equipped with an overvoltage protection circuit. CNT-ELEK is responsible for the power management, the experiment control and the data communication to the payload interface. It is located inside the satellite and is part of the payload stack. CNT-ELEK and CNT-EXT are 
connected with a flat cable. CNT-ELEK consists of a measurement arrangement for the samples. For the resistance measurement of the CNT networks a tranceimpedance amplifier was designed (see Fig. 4).

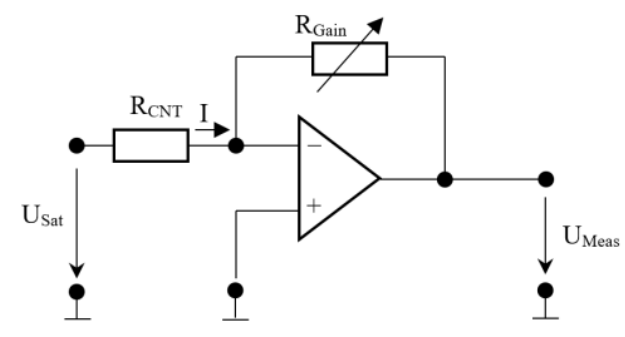

Figure 4: Measurement principle of CiREX based on an inverting amplifier circuit.

The amplification of the signal depends on the supply voltage of the CubSat $\mathrm{U}_{\mathrm{Sat}}$, the gain resistor $\mathrm{R}_{\mathrm{Gain}}$ and the resistance of the CNT network $\mathrm{R}_{\mathrm{CNT}}$. The measured voltage is $\mathrm{U}_{\text {Meas }}$. Their relationship can be described as following:

$$
R_{\text {CNT }}=-\frac{U_{\text {Sat }}}{U_{\text {Meas }}} \cdot R_{\text {Gain }}
$$

Due to the amplifier circuit, it is possible to measure a high resistance. The application of different variable gain resistors ensure a large measuring range from $20 \mathrm{k} \Omega$ up to $100 \mathrm{M} \Omega$. This allows to achieve a consistently high measuring accuracy of $\pm 1 \%$. Furthermore, the temperature is measured and the photocurrent is captured by CNTELEK. The analogue signals are converted by means of a precision 16-bit analogue-to-digital converter. The digital data sets are transferred to the on-board microcontroller of CNT-ELEK.

The microcontroller also handles the communication between the experiment and the payload interface by means of an $\mathrm{I}^{2} \mathrm{C}$ interface.

For CiREX three operation modes are defined: "OFF", "ACTIVE" and "SCIENCE". Therefore, CiREX could be systematically enabled or disabled during the operation phase of SOMP2. In "ACTIVE" mode the system is ready for communication and is able to switch to "SCIENCE" mode. This mode is the most important type of operation for CiREX. In this state, the measurement routine is carried out, which consist of three parts: configuration for the measurement mode (1), measurement (2) and data transmission (3). During the first part the CiREX software receives the measurement parameter from the payload interface. For the measurement mode, two parameters have to be set: the time interval between the measurements points and the number of measurements. After the completion of measurements, the experiment leaves the "SCIENCE" mode and is ready for further communication. All data is saved in the flash memory of the microcontroller and transmitted to the payload interface on request. Due to the measuring algorithm and structure of the analogue circuitry, the minimum measuring interval is $15 \mathrm{~s}$. The data rate and the number of captured orbits depend on the set parameters and are limited by the installed non-volatile memory. At a measuring interval of $60 \mathrm{~s}$ a total data amount of $5.4 \mathrm{kByte}$ is necessary to capture a whole orbit ( 90 minutes). With the given amount of non-volatile memory 35 orbits may be captured continuously before the data has to be transferred to the payload interface. The power consumption of the whole system is about $140 \mathrm{~mW}$ in the active and science mode.

Table 1: Important data of CiREX. 


\begin{tabular}{l|l}
\hline \multicolumn{2}{c}{ Payload experiment CiREX } \\
\hline Mass & CNT-EXT: 8g \\
& CNT-ELEK: 24g \\
Size & CNT-EXT: 23 x $85 \mathrm{~mm}^{2}$ \\
& CNT-ELEK: $83 \times 79 \mathrm{~mm}^{2}$ \\
Power consumption & $>140 \mathrm{~mW}$ \\
Measuring interval & $>15 \mathrm{~s}$ \\
Amount of data per orbit & $5.4 \mathrm{kByte}$ (for $60 \mathrm{~s}$ ) \\
Memory capacity & 35.4 orbits (for $60 \mathrm{~s})$ \\
\hline
\end{tabular}

This flexible measuring principle enables an adaptation of the mission from the ground station. The delay between two measured values can be adjusted. So, either one orbit can be investigated in detail or the degradation process can be observed over several orbits.

Due to the small and standardised size, it is very easy to adapt CiREX to other CubeSat's or experimental platforms. Hence, it will be interesting to place CiREX on different CubeSat's with different orbits in the future. Thus, the degradation process of CNTs in various orbits can be observed and different environmental models can be involved to the evaluation process.

\subsection{Sample preparation for CiREX and ground validation tests}

Identically structured samples have been used for CiREX and the ground validation test. The substrate material for all samples is $\mathrm{Al}_{2} \mathrm{O}_{3}$ (size: $20 \times 3 \mathrm{~mm}^{2}$ ). Gold contacts are printed first followed by the printing of the CNTs. For this step, two different printing processes were used: screen-printing (1) and aerosol jet printing (2).

The screen printing procedure is an easy way to print thick layers on different surfaces. Therefore, we dispersed a multiwalled carbon nanotube/resin (ME) material (by the company INVENT GmbH) into a screen printable paste. This raw material contains epoxy and Baytubes C150HP (by former Bayer MaterialScience) [14]. These MWNTs have a diameter of $13 \mathrm{~nm}$ and a length of more than one $\mu \mathrm{m}$. The layer thickness is up to $27 \mu \mathrm{m}$.

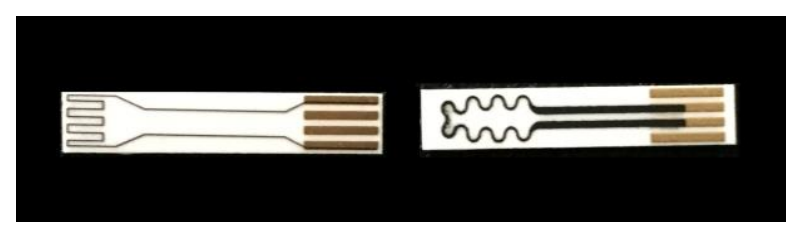

Figure 5: Different types of sample preparation: aerosol jet printed MWNT (left) and screen printed MWNT (right).

For the second process, CNTs were dispersed in water. SWNTs and MWNTs were used for this ink. The SWNT are Tuballs $^{\mathrm{TM}}$ [15] with a diameter of $1.7 \mathrm{~nm}$ and a length of more than $5 \mu \mathrm{m}$. Furthermore, the MWNT are Nanocyl7000 ${ }^{\mathrm{TM}}$ [16] with a diameter of $9.5 \mathrm{~nm}$ and a length of more than $1.5 \mu \mathrm{m}$. Then, the ink is encouraged by ultrasonic vibrations to form an aerosol. This aerosol ink is applied in a defined way by a carrier gas. This process is a non-contact direct-writing technology. The layers reach a thickness up to $8 \mu \mathrm{m}$.

All types of CNTs are commercial products manufactured by a CVD process.

\section{GROUND VALIDATION TEST - SOLAR RADIATION}




\subsection{Test setup and test facility}

The Complex Irradiation Facility at the DLR Bremen [17] is equipped with three light sources to simulate the electro-magnetic radiation from the sun ranging from the vacuum ultra violet (VUV) up to the IR wavelengths. Two corpuscular sources are also available, i.e. a proton and an electron accelerator. The light sources are an ArgonVUV-source [18], a deuterium lamp and a solar simulator with a Xenon lamp. The spectral distribution of the irradiance is shown in Figure 6. Standardized spectra serve as a comparison to the lamps spectra. These spectra are the standard solar spectrum ASTM-490 (sun spectra $>119.5 \mathrm{~nm}$ ) and the Gueyrmard standard (Sun spectrum up to $450 \mathrm{~nm}$ ).

The Argon-VUV-source is a point-light source, which produces light in a board wavelength range from $40 \mathrm{~nm}$ to $410 \mathrm{~nm}$ with a relatively high intensity below $110 \mathrm{~nm}$ in comparison with the Sun's spectrum (see Figure 6). The light is generated by the transition of electrons belonging to excited gas atoms $(98.5 \%$ Argon, $1 \%$ Krypton, and $0.5 \%$ Helium) into their ground state. The excitation occurs by bombardment of the gas molecules with an electron beam inside a vacuum chamber. The gas is injected through a nozzle into the chamber with an adjustable flow rate with the maximum calibrated setting of 1200 standard cubic centimetre per minute $(\mathrm{sccm})$. The main part of the gas is pumped out through a tube below the nozzle with a screw pump. The rest is frozen out onto $\mathrm{Cu}$-baffles which enclose the plasma spot. These baffles are connected with the two stages of the cold heat of a cryogenic pump and kept at cryogenic temperatures.

The deuterium lamp is an electrode excited gas (deuterium) discharge lamp which describes a point-light source too. The discharge arc between the electrically heated cathode and the ring-shaped anode is guided through an aperture of $1 \mathrm{~mm}$ diameter. The light intensity is calibrated for an anode current of $1.8 \mathrm{~A}$ (see Figure 6 ).

The stability of the Argon-VUV-source and the deuterium lamp can be observed with UV-sensitive photodiodes. The first one can be inserted into the light cone of the deuterium lamp inside the irradiation chamber, since the intensity decreases over the lifetime of the lamp. The second one is located at the second light outlet of the VUVsource.

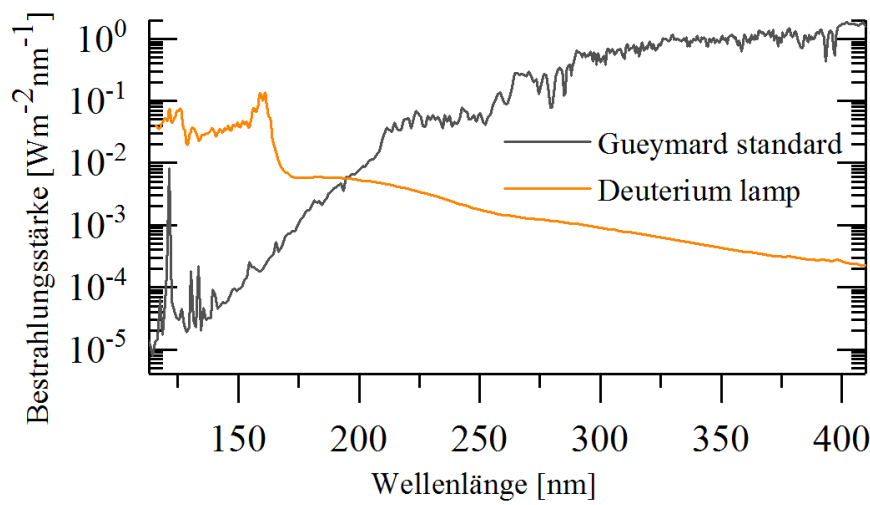

(a) 


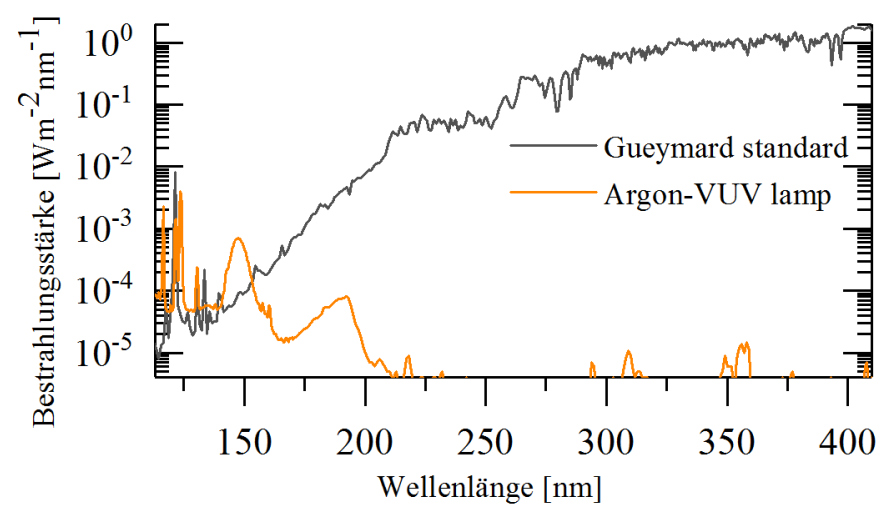

(b)

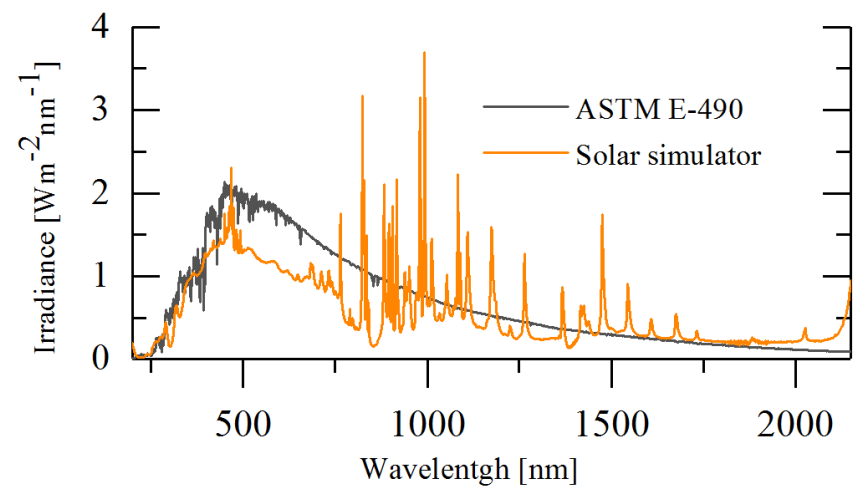

(c)

Figure 6: Spectral distribution of the solar light sources (a) deuterium lamp (b) Argon-VUV jet lamp (c) solar simulator.

For our experiments, we performed three different test scenarios. These tests are listed below (see Table 2).

Table 2: Test setup solar irradiation with different light sources

\begin{tabular}{lll}
\hline Test & Light source & Duration \\
\hline 1 & Solar simulator & 75 min (in addition 45 min break) \\
\hline 2 & $\begin{array}{l}\text { Argon-VUV lamp / Deuterium } \\
\text { lamp }\end{array}$ & 75 min (in addition 45 min break) \\
\hline 3 & $\begin{array}{l}\text { Solar simulator/ Argon-VUV } \\
\text { lamp/ Deuterium lamp }\end{array}$ & 75 min (in addition 45 min break) \\
\hline
\end{tabular}

\subsection{Results and Discussion}

For the investigation of CNTs under different light sources, the initial state of the samples has to be examined. Under atmosphere, CNTs are relatively sensitive to gas exposure and molecules can be physisorbed or chemisorbed at the tubes structure. The exact understanding of these interactions is still unknown. Zhao et al. [19] simulate the adsorption of various gas molecules. He pointed out that the effects of atmospheric gases like $\mathrm{N}_{2}, \mathrm{CO}_{2}$ and $\mathrm{H}_{2} \mathrm{O}$ are relatively weak. Furthermore, he indicates that oxygen has a dominating influence. Therefore, Rajavel et al. [20] describes three main processes for the interaction of oxygen with CNTs: physisorption of molecules at e.g. carboxyl functional sites (1), physisorption on the graphitic tube structure (2) and chemisorption on defects (3). The density functional theory simulations calculated the interaction energy for these bindings to: $-0.753 \mathrm{eV}(1) ;-0.551 \mathrm{eV}$ (2) and $-3.381 \mathrm{eV}$ (3) [20]. 
The samples were exposed to vacuum without any pre-heating process. After this exposure a vacuum initialized desorption process started. In vacuum, there is less interaction of molecules with the tube and functionalisation groups at the tubes surface. The resistance ratio $\left(R / R_{0}\right)$ indicates the changes of the resistance $R$ relative to the resistance $R_{0}$ before the experiment starts. The ratio increases up to $\mathrm{R} / \mathrm{R}_{0} \approx 1.08$ in vacuum (for $\mathrm{SW}$ samples). This vacuum desorption process is a weak process, which could only remove molecules with small bindings energies. As a result, some molecules remain at the tubes surface before the irradiation starts.

Therefore, the samples were stored for more than three hours in vacuum to ensure that this vacuum indicated desorption process is completed and the resistance is stable. This process was monitored by the resistance measurement. After this, the samples were irradiated by the solar light simulator (see Fig.7).

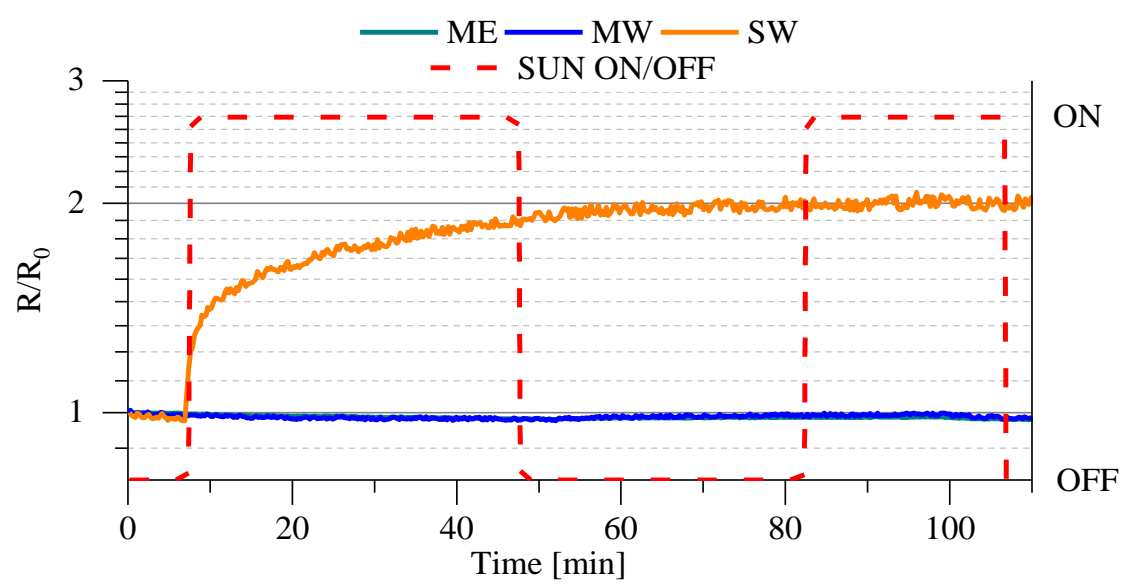

Figure 7: Electrical behaviour of SW, MW and ME under solar light (Xenon lamp; wavelength 200 up to $2150 \mathrm{~nm}$ ).

The highest changes in the resistance were measured for the single-walled (SW) samples. The resistance increases up to $\mathrm{R} / \mathrm{R}_{0} \approx 2$. Even, A. Tchernatinsky et al. [21] observed the increase of the resistance by two orders of magnitude for individual SWNT at $10^{-6}$ Torr after one hour as compared those, which were exposed to $\mathrm{O}_{2}$. For SWNT mats, an $15 \%$ increase of the resistance was observed [21]. In his theoretical study he pointed out that shift of the Fermi level near the top of the valence band causes the electrical sensitivity of the adsorption of $\mathrm{O}_{2}$ molecules [21]. Furthermore, it is known that this effect can change the behavior of the tubes from semiconducting to metallic if the bandgap is small enough [10].

Due to the similarity of our experiment and the results from Tchernatinsky et al. [21] it can be assumed that the desorption process of molecues represents the changes of the electrical resistance. These effects are well-known for new sensor concepts based on carbon nanotubes [22]-[24]. The physisorption of molecules is the result of the weak binding forces on the tubes surface. However, small energies can resolve these bindings. The solar light source heats the samples up to nearly $50{ }^{\circ} \mathrm{C}$ and accelerates the thermal desorption process. After an irradiation of 75 min, the resistance becomes nearly stable. Due to the typical curve progression can be assumed, that irradiation with the Xenon-lamp does not cause no significant damage to the structure of the tubes.

For the MWs, a different behaviour is observed. After the storage in vacuum, the resistance ratio increases up to $\mathrm{R} / \mathrm{R}_{0} \approx 1.02$. During irradiation with solar light only slight variations are observed. These changes are in agreement with the temperature changes of the samples. In general, the morphology and the surface modification of CNTs can substantially influence the reaction kinetics in different environments. The Nanocyl MWNTs have a large number of tubes and defects, which causes a different sensitivity for oxygen in comparison to SWNTs.

Similar to the MWNT, a small temperature depended behaviour of the resistance ratio was observed for the ME. However, there are no significant changes under solar light. One reason for this behaviour is the epoxy, which covers the surface of the tubes. As a result, an interaction between tube and oxygen is prevent.

Calculations suggest that the interaction between the CNTs and the molecular oxygen is weak. The dissociative adsorption of oxygen atoms on defects and edges is much stronger [25]. That implies that different wavelength could 
have a different impact on the tubes structure. For the second tests, the samples were irradiated with ultraviolet light (UV) and vacuum ultraviolet light (VUV).

Also, in this test, the biggest changes in resistance were measured for SW (see Fig. 8). The resistance increases up to $\mathrm{R} / \mathrm{R}_{0} \approx 2.3$ after $75 \mathrm{~min}$ of irradiation. Furthermore, the resistance ratio does not become stable at the magnitude of two and is continuing to increase. Besides the physisorbed molecules, chemisorbed molecues are bonded to the tube structure [25]. The removal of this chemisorbed molecules e. g. oxygen can damage the structure surrounding the atom, because $\mathrm{C}-\mathrm{O}$ bonds are more stable than $\mathrm{C}-\mathrm{C}$ bonds [25]. Depending on the type of binding the desorption energy varies. If the energy is high enough the UV light brakes these bindings and increases the defect density of the tubes.

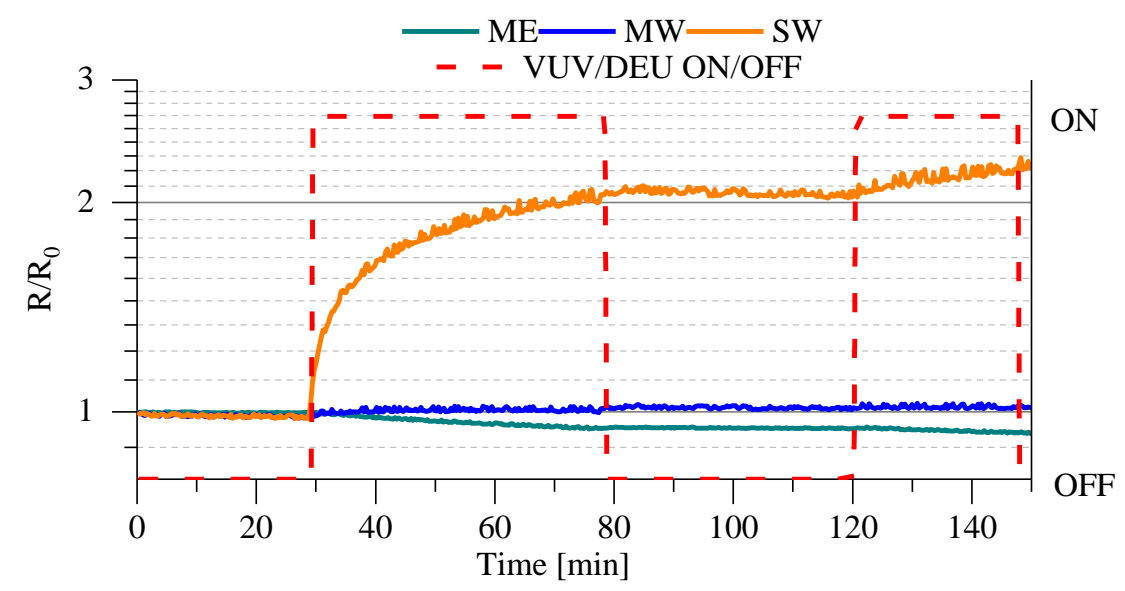

Figure 8 : Electrical behaviour of SW, MW and ME under UV light (deuterium lamp and Argon-VUV lamp; wavelength 40 up to $410 \mathrm{~nm})$.

The MW resistance ratio is nearly stable under UV-light. It can be assumed that the removal of strong oxygen bindings cause also defects in the MWNT structure. Due to their multi-tube structure, the electrical resistance of the MWNTs are non-sensitive for small structural changes in the outer tubes. As long as intact tubes exist, the electrical conductance path is still active due to the multi-tube structure. As a result, the electrical resistance is nearly stable despite the small structural defects. Therefore, MWNT are consider as more stable than SWNT. In addition, the UV light source induces a smaller amount of heat in the samples like the Xenon lamp and so does not influence the sample temperature.

The resistance ratio of the composite material (ME) decreases under UV light. Photo-induced depolymerisation and erosion processes results in a lower resistance connection between the CNTs and changes the electrical properties of the composite sample [26, 27]. These interactions between the matrix material and the carbon nanotubes are known and is part of new sensor concepts $[26,28]$. Therefore, it can be assumed that these effects are responsible for these changes for the electrical properties.

For the last test (see Fig. 9), all three light sources were used. The behaviour of the resistance ratio of the $S W$ is similar to the previous test. Therefore, the $\mathrm{R} / \mathrm{R}_{0}$ does not stagnate around factor two. The resistance ratio increases up to $\mathrm{R} / \mathrm{R}_{0} \approx$ 2.5. Thus, we were able to measure a higher and faster increase of the resistance with all three light sources. Furthermore, the decrease of the resistance of the ME samples seems to be faster in this test. This effect is still not clear and has to be investigated in detail. It could be accepted that the heat input of the Xenon lamp, which leads to an acceleration of the molecules desorption and a degradation process. Furthermore, it may be assumed that the degradation process of the SW tubes is slowed down, if all surface bindings are removed. 


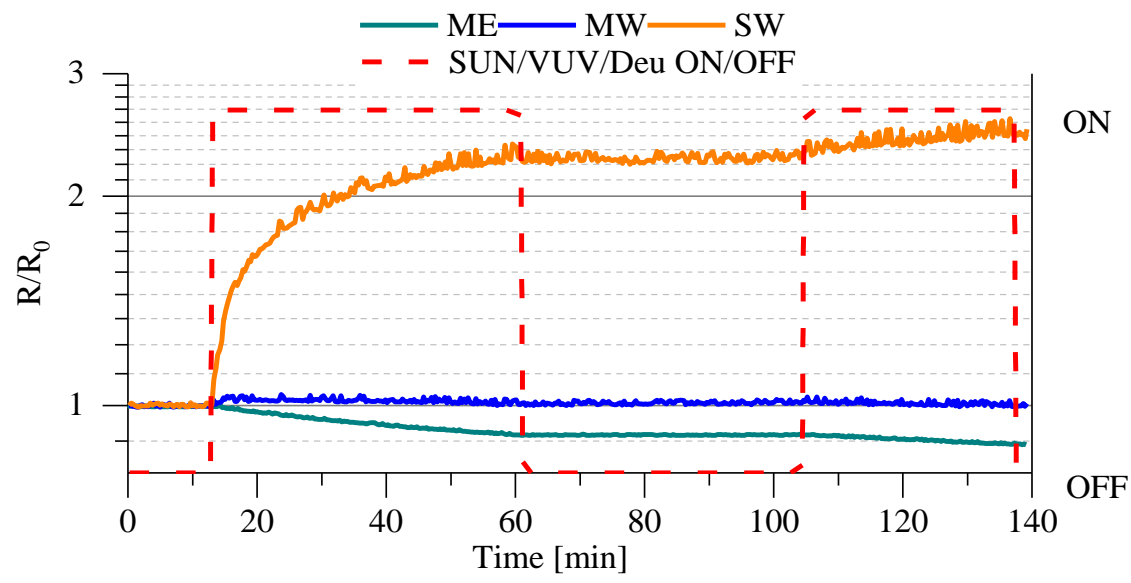

Figure 9: Electrical behaviour of SW, MW and ME under solar and UV light (xenon lamp, deuterium lamp and argon jet lamp; wavelength 40 up to $2500 \mathrm{~nm}$ ).

The behaviour of the resistance ratio can only indicate the degradation process. Further analytical measurement techniques are necessary to investigate the degradation process under solar light. Therefore, Raman measurements with a laser wavelength of $532 \mathrm{~nm}$ were performed. The G-band $\left(\sim 1590 \mathrm{~cm}^{-1}\right)$ corresponds to the planar vibration of the carbon atoms. Furthermore, the D-band $\left(\sim 1340 \mathrm{~cm}^{-1}\right)$ is a result of substitutional heteroatoms, vacancies, grain boundaries or defects and provides important information regarding the degree of crystallinity of CNT. Hence, the intensity ratio $\mathrm{I}(\mathrm{D}) / \mathrm{I}(\mathrm{G})$ of these modes is an important parameter for materials analysis. If $\mathrm{I}(\mathrm{D}) / \mathrm{I}(\mathrm{G})$ is $s m a l l$, the molecules are well organized in the hexagonal lattice. Consequently, this method can be used to obtain structural information about the CNT sample.[29]

For the pristine SW samples the intensity ratio area $\mathrm{I}(\mathrm{D}) / \mathrm{I}(\mathrm{G})$ was calculated to be $0.12 \pm 0.02$ (see Fig.10). After the first test (irradiation with solar light), an intensity ratio of $0.14 \pm 0.02$ was determined. There was no clear increase of the resistance ratio after the solar irradiation. However after test two and three the $\mathrm{I}(\mathrm{D}) / \mathrm{I}(\mathrm{G})$ raised up to $0.18 \pm 0.02$ and $0.18 \pm 0.02$ with a clear increase of the D-Band $\left(\sim 1340 \mathrm{~cm}^{-1}\right)$. Therefore, it can be stated that the defect density in the tubes increased after the combined light irradiation. The tubes hexagonal structure has been damaged.

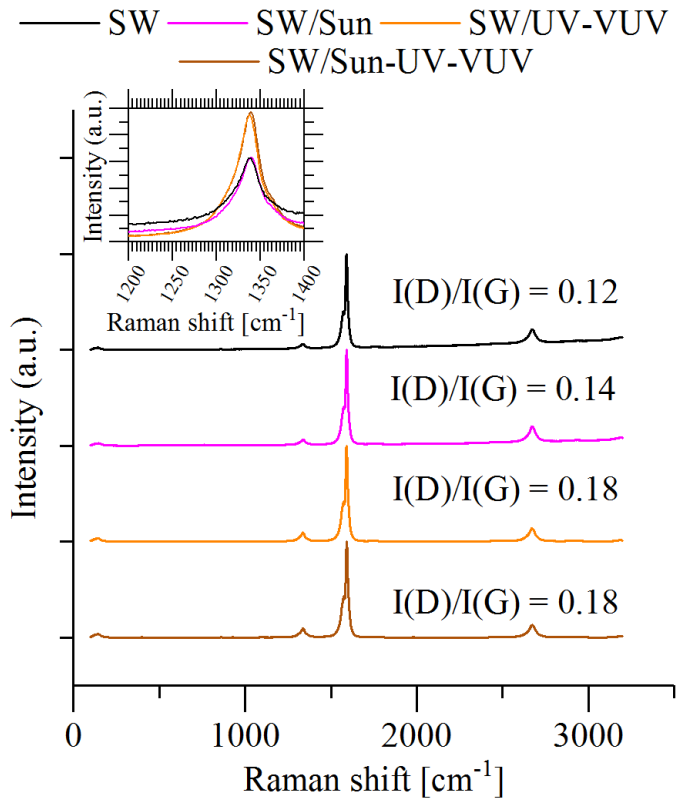

(a)

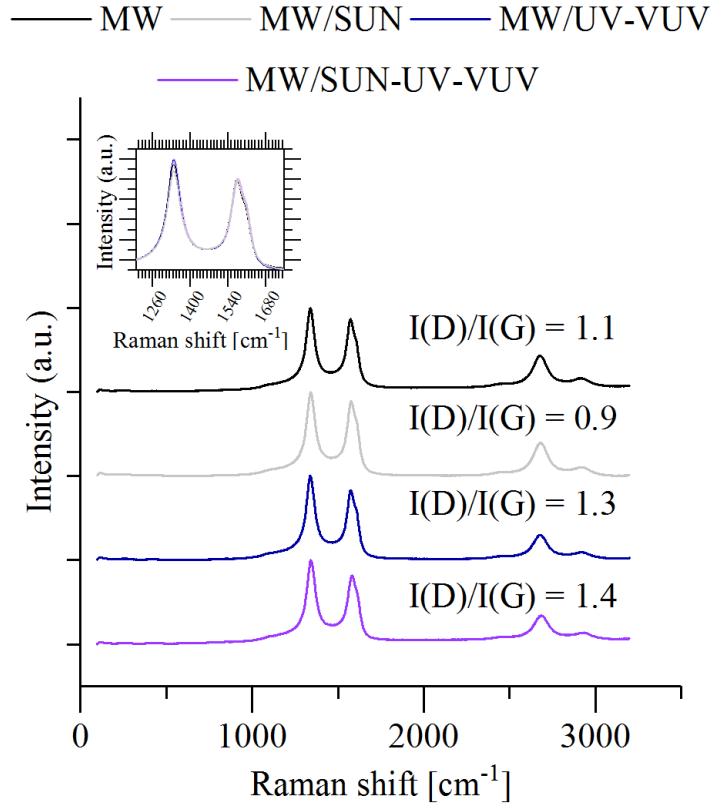

(b) 


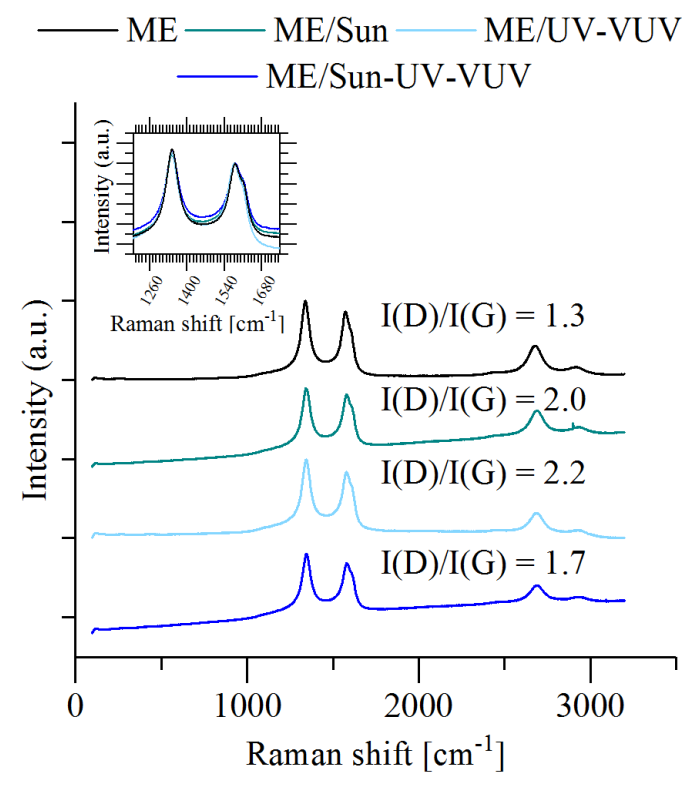

(c)

Figure 10: Raman spectra of (a) SW, (b) MW, (c) ME under irradiation with the xenon lamp; with Argon-VUV lamp and deuterium lamp and all three light sources.

Also for the MWNT the D- as well as the G-band are present. However, the initial ratio of $1.1 \pm 0.2$ is different compared to the SWNT as a result of the increased disordered parts of the sample. After solar light irradiation, the intensity ratio decreases to $0.9 \pm 0.2$. Whereas the irradiation with UV and combined solar and UV light leads to increased ratios of $1.3 \pm 0.2$ and $1.4 \pm 0.2$ respectively (see Fig. 10(b)). The defect density for MWNT is even higher than for SWNT. Small changes in the defect density are difficult to measure. The results of the measurements show a small increase of the intensity ratio for the samples irradiated with UV-VUV and solar light-UV-VUV, which is an indication for a degradation process. At the moment, it is not clear why the $\mathrm{I}(\mathrm{D}) / \mathrm{I}(\mathrm{G})$ decreases after the irradiation with the Xenon lamp. Perhaps, solar light initiated healing and rearrangement effects of carbon atoms.

For the composite material an increased intensity ratio for all light sources up to $2.0 \pm 0.2 ; 2.2 \pm 0.2$ and $1.7 \pm 0.2$ (see Fig. 10(c)) was observed. The weight percentage of the carbon nanotubes in the composite is high (up to 50\%). Therefore, the signal intensity of Raman spectra of the CNT are much stronger and so the spectra of the epoxy material cannot be seen. In our measurement, the tubes undergo small changes in their structure. Wide parts of the surfaces are covered with epoxy. Thus, the physisorption and chemisorption processes are not activated under electromagnetic irradiation.

The behaviour of the epoxy is sensitive to solar light, especially UVA or UVB [30]. It can be reasonably assumed that irradiation with UV-VUV and solar light-UV-VUV change the structure of the epoxy and consequently the interaction with the tubes. This structural change could cause the decrease of the resistance ratio. 

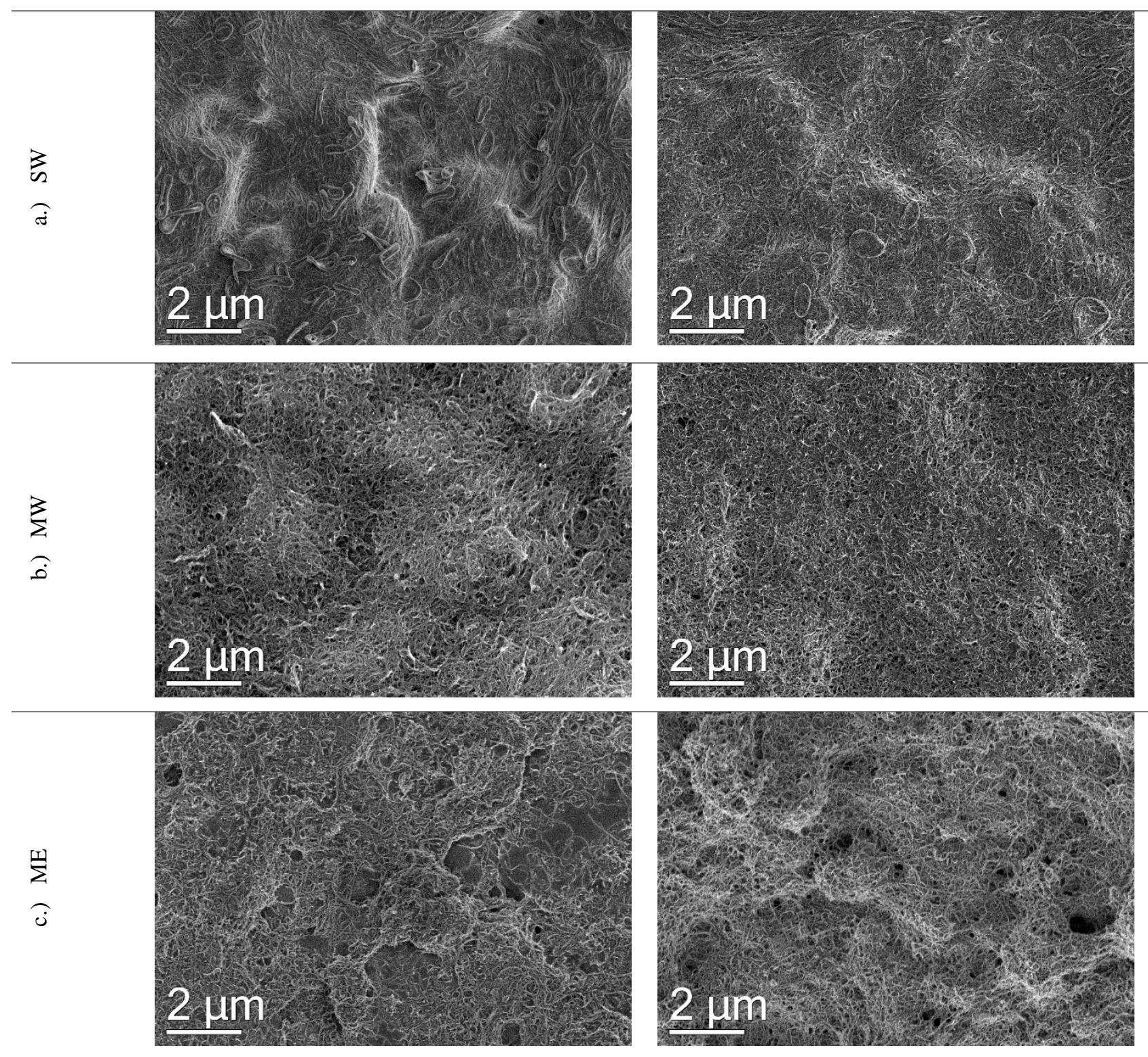

Figure 11: SEM micrographs of different types of CNTs: (left) without irradiation and after irradiation with all light sources (right).

Scanning electron microscopy (SEM) imaging is a well-established method for characterising the morphological changes of the CNTs after light irradiation. Such morphological changes, due to tube damaging, are known in case of low-energy ion irradiation or oxidation processes $[9,31,32]$. It was discovered that CNTs form e.g. thickened nanorods, if the number of defects, e.g. dislocations, is high enough after ion irradiation [33]. As a result of such extreme damage, the tubes change their morphology significantly.

For the present study, SEM micrographs of the samples irradiated with various light sources are shown in Fig. 11. In addition to the Raman measurements, SEM imaging of the samples do not indicate extreme morphological changes, which are known e.g. in case of low-energy ion irradiation. Regarding the CNTs, however, we could not detect extraordinary structural changes. This is a clear indication that the electromagnetic light sources cause only a small increase of the defect density and small structural changes in the tube structure. The observations above are in full agreement with the electrical measurements and the Raman scattering spectroscopy analysis. 


\section{CONCLUSION}

In summary, we designed a small material experiment (CiREX) for CubeSat. The dimensions are $23 \times 85 \mathrm{~mm}^{2}$ (CNTEXT) with a weight of $8 \mathrm{~g}$. It is a completely autonomous experiment, which can be initialised by the satellites payload interface. The measurement mode can be adapted during the mission.

The experiment focuses on the degradation behaviour of different types of CNT. Therefore, the samples are directly exposed to space environment and the electrical resistance of CNT networks is measured. Printed networks of SWNT, MWNT and MWNT/epoxy are available.

CiREX requires a series of ground validation tests which evaluate the CNT behaviour under solar light. Accordingly, three different light sources (Xenon lamp, Argon-VUV lamp and deuterium lamp) were used to simulate the solar spectra up to the deep VUV light of $40 \mathrm{~nm}$. Our measurements show three different interactions of the tubes with oxygen especially for SWNT. The molecular interaction with the tubes causes an increase in resistance during the evacuation process. The desorption process is activated under solar light (Xenon lamp). There was no clear evidence for structural changes of the tubes. Chemisorbed bindings can be cracked through UV and VUV light irradiation. As a result, we were able to measure an increased resistance and a changed defect density.

The surface of the CNT composite is covered with epoxy leading to a reduced interaction with surrounding molecules. However, the depolymerisation process of the epoxy can also change the electrical behaviour under UV light irradiation.

The irradiation with a solar light spectrum changed the electrical resistance of the tubes due to the interaction with oxygen. Especially SWNT are sensitive to oxygen. Overall, it can be assumed that CiREX can track these changes in the initial phase of the mission.

\section{ACKNOWLEDGMENT}

This work was partly funded by the Deutsches Zentrum für Luft- und Raumfahrt (DLR) on behalf of the Bundesministeriums für Wirtschaft und Energie (BMWI, FKZ 50YB1409, FKZ 50RU1202). Support by the Ion Beam Center (IBC) at the Helmholtz-Zentrum Dresden-Rossendorf is gratefully acknowledged.

\section{REFERENCES}

[1] M. F. L. De Volder, S. H. Tawfick, R. H. Baughman, and A. J. Hart, 'Carbon Nanotubes: Present and Future Commercial Applications', Science, vol. 339, no. 6119, pp. 535-539, Feb. 2013.

[2] R. H. Baughman, A. A. Zakhidov, and W. A. de Heer, 'Carbon Nanotubes--the Route Toward Applications', Science, vol. 297, no. 5582, pp. 787-792, Aug. 2002.

[3] S. Rawal, J. Brantley, and N. Karabudak, 'Development of carbon nanotube-based composite for spacecraft components', in 2013 6th International Conference on Recent Advances in Space Technologies (RAST), 2013, pp. $13-19$.

[4] L. Pourzahedi, P. Zhai, J. A. Isaacs, and M. J. Eckelman, 'Life cycle energy benefits of carbon nanotubes for electromagnetic interference (EMI) shielding applications', J. Clean. Prod., vol. 142, Part 4, pp. 1971-1978, Jan. 2017.

[5] A. R. Hopkins, A. C. Labatete-Goeppinger, H. Kim, and H. A. Katzman, 'Space survivability of carbon nanotube yarn material in low Earth orbit', Carbon, vol. 107, pp. 77-86, Oct. 2016.

[6] A. R. Adhikari, M. B. Huang, H. Bakhru, S. Talapatra, P. M. Ajayan, and C. Y. Ryu, 'Effects of proton irradiation on thermal stability of single-walled carbon nanotubes mat', Nucl. Instrum. Methods Phys. Res. Sect. B Beam Interact. Mater. At., vol. 245, no. 2, pp. 431-434, Apr. 2006.

[7] B. LI, Y. FENG, K. DING, G. QIAN, X. ZHANG, and Y. LIU, 'Effect of electron beam irradiation on multiwalled carbon nanotubes', Trans. Nonferrous Met. Soc. China, vol. 24, no. 3, pp. 764-769, Mar. 2014.

[8] F. Banhart, 'Irradiation effects in carbon nanostructures', Rep. Prog. Phys., vol. 62, no. 8, pp. 1181-1221, Aug. 1999.

[9] E. Abbe et al., 'Electrical behaviour of carbon nanotubes under low-energy proton irradiation', J. Nucl. Mater.

[10] M. Grujicic, G. Cao, A. M. Rao, T. M. Tritt, and S. Nayak, 'UV-light enhanced oxidation of carbon nanotubes', Appl. Surf. Sci., vol. 214, no. 1, pp. 289-303, May 2003.

[11] C. O. Lee, E. Najafi, J. Y. Kim, S.-H. Han, T. Lee, and K. Shin, 'Effects of Protons, Electrons, and UV Radiation on Carbon Nanotubes', in Polymer Durability and Radiation Effects, vol. 978, M. C. Celina and R. A. Assink, Eds. Washington, DC: American Chemical Society, 2007, pp. 232-252.

[12] B. Li, Y. Feng, K. Ding, G. Qian, X. Zhang, and J. Zhang, 'The effect of gamma ray irradiation on the structure 
of graphite and multi-walled carbon nanotubes', Carbon, vol. 60, pp. 186-192, Aug. 2013.

[13] C. Mikó, M. Milas, J. W. Seo, R. Gaál, A. Kulik, and L. Forró, 'Effect of ultraviolet light irradiation on macroscopic single-walled carbon nanotube bundles', Appl. Phys. Lett., vol. 88, no. 15, p. 151905, Apr. 2006.

[14] A. Bayer MaterialScience, 'Data sheet Baytubes ${ }^{\circledR}$ C150P', Ed. 2009Y02Y24, 2009.

[15] TUBALL ${ }^{T M}$ Single Wall Carbon Nanotubes. .

[16] NC7000 ${ }^{T M}$ - Nanocyl. .

[17] T. Renger, M. Sznajder, A. Witzke, and U. Geppert, 'The Complex Irradiation Facility at DLR-Bremen', in Third International Symposium on Solar Sailing, Glasgow, Schottland, 2013.

[18] M. Sznajder, T. Renger, A. Witzke, U. Geppert, and R. Thornagel, 'Design and performance of a vacuum-UV simulator for material testing under space conditions', Adv. Space Res., vol. 52, no. 11, pp. 1993-2005, Dec. 2013.

[19] J. Zhao, A. Buldum, J. Han, and J. P. Lu, 'Gas molecule adsorption in carbon nanotubes and nanotube bundles', Nanotechnology, vol. 13, no. 2, pp. 195-200, Apr. 2002.

[20] K. Rajavel, M. Lalitha, J. K. Radhakrishnan, L. Senthilkumar, and R. T. Rajendra Kumar, 'Multiwalled Carbon Nanotube Oxygen Sensor: Enhanced Oxygen Sensitivity at Room Temperature and Mechanism of Sensing', ACS Appl. Mater. Interfaces, vol. 7, no. 43, pp. 23857-23865, Nov. 2015.

[21] A. Tchernatinsky et al., 'Adsorption of oxygen molecules on individual single-wall carbon nanotubes', J. Appl. Phys., vol. 99, no. 3, p. 034306, Feb. 2006.

[22] P. Slobodian, P. Riha, A. Lengalova, P. Svoboda, and P. Saha, 'Multi-wall carbon nanotube networks as potential resistive gas sensors for organic vapor detection', Carbon, vol. 49, no. 7, pp. 2499-2507, Jun. 2011.

[23] I. Sayago et al., 'Carbon nanotube networks as sensitive layers for resistive gas sensor applications', Nanopages, vol. 8, no. 1, pp. 15-26, Jun. 2013.

[24] C. Cantalini, L. Valentini, I. Armentano, L. Lozzi, J. M. Kenny, and S. Santucci, 'Sensitivity to NO2 and crosssensitivity analysis to NH3, ethanol and humidity of carbon nanotubes thin film prepared by PECVD', Sens. Actuators B Chem., vol. 95, no. 1, pp. 195-202, Oct. 2003.

[25] Y. Miyamoto, N. Jinbo, H. Nakamura, A. Rubio, and D. Tománek, 'Photodesorption of oxygen from carbon nanotubes', Phys. Rev. B, vol. 70, no. 23, p. 233408, Dec. 2004.

[26] J. Lobez and T. Swager, 'Radiation detection: resistivity responses in functional poly(olefin sulfone)/carbon nanotube composites.', Angew. Chem. Int. Ed., vol. 49, pp. 95-8, 2010.

[27] T. Nguyen et al., 'Impact of UV irradiation on multiwall carbon nanotubes in nanocomposites: Formation of entangled surface layer and mechanisms of release resistance', Carbon, vol. 116, no. Supplement C, pp. 191-200, May 2017.

[28] C. J. Long et al., 'Giant Surface Conductivity Enhancement in a Carbon Nanotube Composite by Ultraviolet Light Exposure', ACS Appl. Mater. Interfaces, vol. 8, no. 35, pp. 23230-23235, Sep. 2016.

[29] A. C. Ferrari and J. Robertson, 'Interpretation of Raman spectra of disordered and amorphous carbon', Phys. Rev. $B$, vol. 61, no. 20, pp. 14095-14107, May 2000.

[30] G. Zhang, W. G. Pitt, S. R. Goates, and N. L. Owen, 'Studies on oxidative photodegradation of epoxy resins by IR-ATR spectroscopy', J. Appl. Polym. Sci., vol. 54, no. 4, pp. 419-427, Oct. 1994.

[31] A. V. Krasheninnikov and K. Nordlund, 'Irradiation effects in carbon nanotubes', Nucl. Instrum. Methods Phys. Res. Sect. B Beam Interact. Mater. At., vol. 216, pp. 355-366, Feb. 2004.

[32] S. Suzuki, 'Low-Energy Irradiation Damage in Single-Walled Carbon Nanotubes', 2011.

[33] S. Honda et al., 'Transformation of multiwalled carbon nanotubes to amorphous carbon nanorods under ion irradiation', Jpn. J. Appl. Phys., vol. 53, no. 2S, p. 02BD06, Jan. 2014. 